\title{
A histone chaperone, DEK, transcriptionally coactivates a nuclear receptor
}

\author{
Shun Sawatsubashi ${ }^{1,2}$ Takuya Murata, ${ }^{1}$ Jinseon Lim, ${ }^{1}$ Ryoji Fujiki, ${ }^{1}$ Saya Ito, ${ }^{1,2}$ Eriko Suzuki, ${ }^{1,2}$ \\ Masahiko Tanabe, ${ }^{1,2}$ Yue Zhao, ${ }^{1}$ Shuhei Kimura, ${ }^{1}$ Sally Fujiyama, ${ }^{1,2}$ Takashi Ueda, ${ }^{1}$ Daiki Umetsu, ${ }^{1}$ \\ Takashi Ito, ${ }^{3}$ Ken-ichi Takeyama, ${ }^{1}$ and Shigeaki Kato ${ }^{1,2,4}$ \\ ${ }^{1}$ Institute of Molecular and Cellular Biosciences, University of Tokyo, Tokyo 113-0032, Japan; ${ }^{2}$ Exploratory Research for \\ Advanced Technology, Japan Science and Technology Agency, Saitama 332-0012, Japan; ${ }^{3}$ Department of Biochemistry, Nagasaki \\ University School of Medicine, Nagasaki 852-8523, Japan
}

Chromatin reorganization is essential for transcriptional control by sequence-specific transcription factors. However, the molecular link between transcriptional control and chromatin reconfiguration remains unclear. By colocalization of the nuclear ecdysone receptor (EcR) on the ecdysone-induced puff in the salivary gland, Drosophila DEK (dDEK) was genetically identified as a coactivator of EcR in both insect cells and intact flies. Biochemical purification and characterization of the complexes containing fly and human DEKs revealed that DEKs serve as histone chaperones via phosphorylation by forming complexes with casein kinase 2. Consistent with the preferential association of the DEK complex with histones enriched in active epigenetic marks, dDEK facilitated H3.3 assembly during puff formation. In some human myeloid leukemia patients, DEK was fused to CAN by chromosomal translocation. This mutation significantly reduced formation of the DEK complex, which is required for histone chaperone activity. Thus, the present study suggests that at least one histone chaperone can be categorized as a type of transcriptional coactivator for nuclear receptors.

[Keywords: DEK; acute myeloid leukemia; histone chaperone; ecdysone receptor; coactivator; histone variant H3.3] Supplemental material is available at http://www.genesdev.org.

Received August 26, 2009; revised version accepted November 30, 2009.

Chromatin structure is reorganized during gene activation through chromatin remodeling and epigenetic modification (Henikoff 2008; Jiang and Pugh 2009). Through these processes, nucleosomal DNA becomes accessible to sequence-specific transcription factors, facilitating their stable binding at specific sites in target gene promoters (Cairns 2009). Chromatin reconfiguration supporting transcriptional initiation and subsequent premRNA elongation requires a number of functionally distinct regulatory complexes (Narlikar et al. 2002; Roeder 2005; Rosenfeld et al. 2006). One such class is an ATP-dependent chromatin remodeling complex. This class of complexes uses ATP hydrolysis to directly rearrange nucleosomal arrays in a noncovalent manner by sliding and transferring histone octamers associating with chromosomal DNA (Kouzarides 2007; Morrison and Shen 2009). Another class of regulatory complexes can be categorized as histone-modifying enzymes, which epige-

${ }^{4}$ Corresponding author.

E-MAIL uskato@mail.ecc.u-tokyo.ac.jp; FAX 81-3-5841-8477.

Article published online ahead of print. Article and publication date are online at http://www.genesdev.org/cgi/doi/10.1101/gad.1857410. netically modify histone proteins (Borrelli et al. 2008; Sims and Reinberg 2008). These enzymes covalently supply reversible epigenetic marks (acetyl groups, methyl groups, and phosphates) at specific residues on histone tails. Combinations of histone modifications generate a "histone code" to direct chromatin configuration in surrounding chromatin areas (Strahl and Allis 2000; Kouzarides 2007). Moreover, the eviction and reassembly of histone octamers at transcribing gene loci are evident during chromatin reconfiguration, and require a third class of nucleosomal regulators: histone chaperones (De Koning et al. 2007; Park and Luger 2008). In this regard, histone chaperones are believed to transcriptionally coregulate the function of sequence-specific regulators because core histones are mobilized during the transcription and elongation process (Adkins et al. 2004; Adkins and Tyler 2006). Specific deposition of histone variants like histone $\mathrm{H} 3.3$ is also well documented in transcriptionally active loci in Drosophila cells (Mito et al. 2005; Schwartz and Ahmad 2005; Henikoff 2008), but the histone chaperones involved in this deposition have not been identified. The exact form of histone chaperone units and their mode of function appear diverse. However, 
the role of each histone chaperone in the processes of transcriptional control by sequence-specific regulators is poorly understood.

An insect steroid hormone, ecdysone, induces metamorphosis (Thummel 1996). Like mammalian nuclear receptors (NRs) (Evans 1988; Green and Chambon 1988), nuclear ecdysone receptor (EcR) has been characterized as a ligand-dependent and sequence-specific transcriptional activator, and heterodimerizes with ultraspiracle (USP) to control target gene expression in an ecdysone-dependent manner (Koelle et al. 1991; King-Jones and Thummel 2005). Not surprisingly, key transcriptional coregulators are functionally and structurally conserved from insects to mammals (Bai et al. 2000; Takeyama et al. 2002; Sedkov et al. 2003). This observation suggests that chromatin reconfiguration might be essential for EcRmediated transcriptional control, as observed previously for mammalian NRs. In this respect, ecdysone-induced puff formation in the salivary gland of the fly (Ashburner 1990; Thummel 2002) is a readily observed example of chromatin reorganization induced by NRs. Although the prominent morphological alteration of chromatin structure was initially described decades ago (Ashburner 1967), the molecular basis and the associated regulatory factors are scarcely known. In the present study, we used Drosophila genetic screening to identify regulators supporting chromatin reorganization induced by liganded EcR. We found that a chaperone, Drosophila DEK (dDEK), is colocalized with EcR at the ecdysone-induced puff, and acts as a transcriptional EcR coactivator. Biochemical purification and characterization of fly and human DEK (hDEK) complexes revealed that phosphorylated DEKs associating with casein kinase 2 (CK2) serve as a histone chaperone. Moreover, in a group of acute myeloid leukemia (AML) patients, a mutant hDEK protein is known to be fused with CAN (Soekarman et al. 1992; von Lindern et al. 1992). We found the mutant to be defective in chaperone activity. Thus, the present study suggests that a specific class of histone chaperones serves as a NR coactivator.

\section{Results}

Genetic screening identified dDEK

as an ecdysone-inducible puff-localized factor

To identify a regulator involved in ecdysone-induced puff formation in the salivary gland of Drosophila, we genetically screened candidates from EGFP protein trap library lines (Morin et al. 2001; Buszczak et al. 2007) treated with a synthetic ecdysone: Muristerone A (Mur) (Supplemental Fig. S1A). In the induced puff, several candidate lines colocalizing EGFP with EcR were identified. Among them, two lines-G00131 (Fig. 1A) and CA06616 (Supplemental Fig. S1B; Morin et al. 2001; Buszczak et al. 2007)—were selected for further analysis. The sequence of the flanking genomic DNA and the transcripts from these two lines led us to identify the same gene (Fig. 1B), the Drosophila ortholog (dDEK) of the $h D E K$ oncogene (von Lindern et al. 1992). To characterize endogenous
dDEK expression in the salivary gland, we generated a polyclonal antibody against dDEK (Supplemental Fig. S1C). Staining of polytene chromosomes from wild-type larvae with the antibody showed that dDEK and EcR overlapped on puffs (Supplemental Fig. S2A). Based on the immunofluorescence of polytene chromosomes with anti-Ser5-phosphorylated RNA polymerase II (Pol II) (Weeks et al. 1993), dDEK appeared to be associated with transcriptionally active loci (Fig. 1C). dDEK was seen in the less-compact chromatin interbands (estimated as weak DAPI staining), and its location was the converse of that of histone $\mathrm{H1}$, a marker of condensed chromatin (Fig. 1D; Supplemental Fig. S2B; Kim et al. 2004). These findings suggested that $\mathrm{dDEK}$ was localized in regions of transcriptionally active chromatin.

\section{dDEK is an EcR coactivator}

We examined the possible association of dDEK with EcR in Drosophila S2 cells. dDEK was coimmunoprecipitated with EcR in the absence or presence of Mur (Fig. 2A). Next, physical interaction was tested by a pull-down assay using S-tagged dDEK with EcR recombinant protein prepared in a baculoviral expression system. Ligand-independent association of EcR with dDEK was seen even at high $\mathrm{NaCl}$ concentration (Fig. 2B). Thus, dDEK appeared to be a ligand-independent interactant for EcR.

To determine if dDEK was functionally involved in ecdysone-induced chromatin reorganization, we tested the impact of dDEK in ecdysone-induced gene expression in intact flies. We used an RNAi approach with the GAL4-UAS binary system to knock down endogenous dDEK (Pili-Floury et al. 2004). We established UAS inverted repeat (IR) transgenic strains, carrying fragments of the $d D E K$ gene to form dsRNA to target the dDEK transcript under control of a salivary gland-specific GAL4 driver (sgs3-GAL4) (Stabell et al. 2007). Likewise, either EcR or Taiman (Tai) was knocked down in the salivary glands (Supplemental Fig. S3A). As anticipated, in vitro treatment of wild-type flies' salivary glands with Mur induced expression of EcR target genes (Eip74EF, Eip75B, and $B R-C$ ) located within puff regions (Fig. 2C; Supplemental Fig. S4A; Burtis et al. 1990; Karim and Thummel 1992). However, knockdown of either dDEK (IR-dDEK) or EcR (IR-EcR) resulted in the loss of the Mur response, which was also observed following knockdown of Tai (IRTai) (Fig. 2C; Supplemental Fig. S4A). Taiman, a Drosophila homolog of human AIB1 histone acetyltransferase (HAT), is known to coactivate EcR (Bai et al. 2000). Thus, it appeared that dDEK was a transcriptional EcR coactivator. Using the same knockdown approach with Mur-treated S2 cells (Supplemental Fig. S3B), we verified a significant role of dDEK in EcR-mediated gene induction (Supplemental Fig. S4B).

To directly test if dDEK coactivated the transactivation function of EcR, the coregulator role of $\mathrm{DDEK}$ was tested in a transient luciferase reporter assay (Sawatsubashi et al. 2004). Overexpression of dDEK coactivated EcR in the presence of Mur (Fig. 2D, lanes 3,4), without potentiation of the basal promoter activity (Fig. 2D, lanes 1,2), consistent 

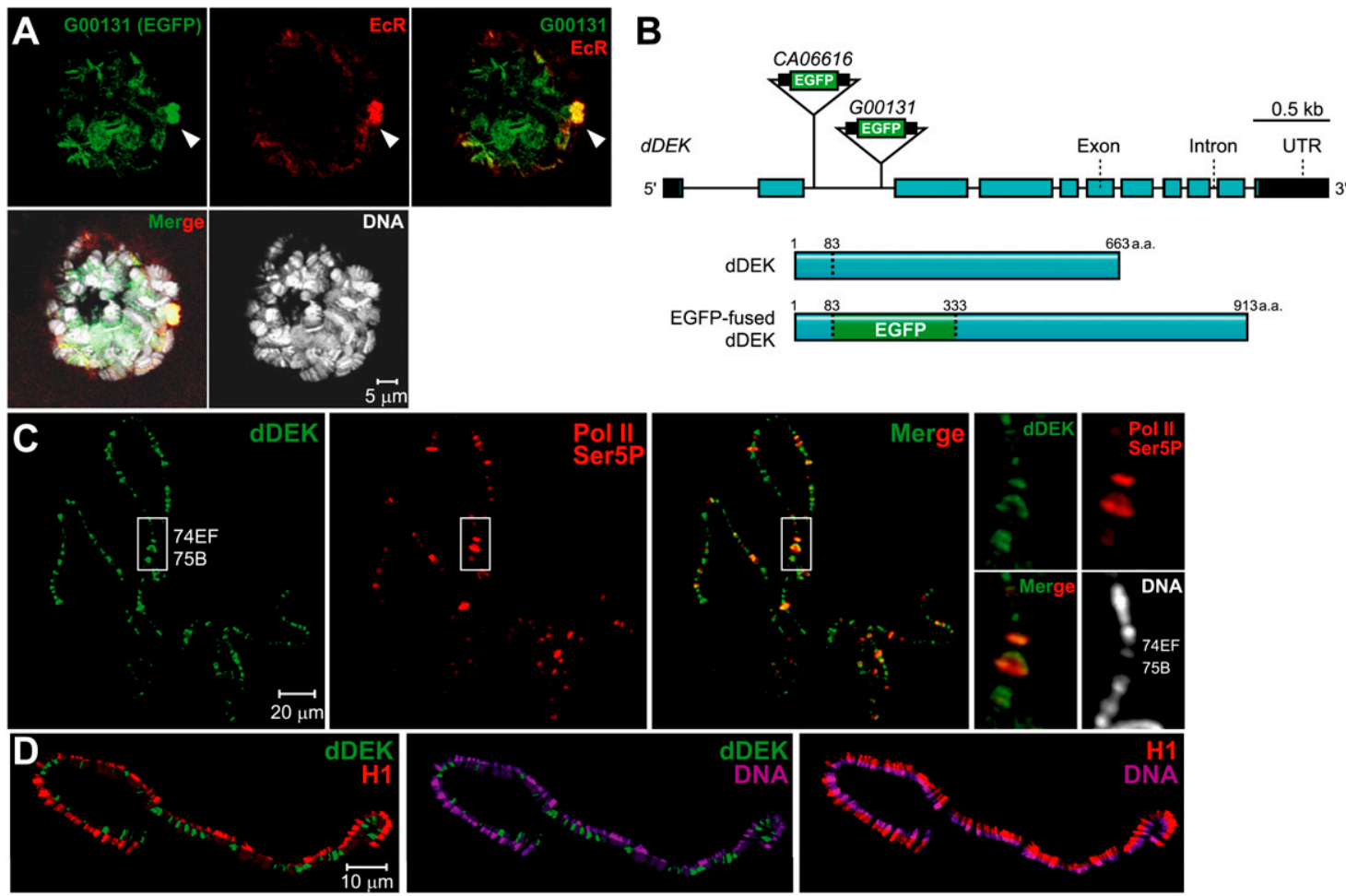

Figure 1. Localization of dDEK within the ecdysone-induced puff. (A) Third instar larval salivary gland nuclei of the G00131 protein trap line in which the EGFP-fused protein (green) colocalizes with the EcR (marked by an anti-EcR antibody, stained red) at ecdysoneinduced puff loci (arrowhead) and interbands on polytene chromosomes (DNA stain, white). (B) Insertion sites of protein trap lines in G00131 and CA06616. (Top) The dDEK transcript fused to the EGFP exon (green box) carried by line G00131 or CA06616. The untranslated region (black), coding regions (blue), and insertion alleles (triangle) are shown. (Bottom) Schematic representation of deduced EGFP-fused dDEK proteins expressed in G00131 is also shown. $(C)$ Immunolocalization of dDEK on wild-type polytene chromosomes. The wild-type polytene chromosome squashes are costained with anti-dDEK (green) and anti-RNA polymerase II Ser5-P (as a maker of puff loci) antibodies (red) and DAPI (DNA stain, white). Endogenous dDEK is enriched at specific polytene chromosome sites, including ecdysone-induced puffs at 74EF and 75B (white box). The right panels present higher-magnification images of the whiteboxed areas. $(D)$ The distributions of dDEK (green) and H1 (as a marker of condensed chromatin, red) are shown in split images, including costaining for DNA (magenta). dDEK primarily associated with interband regions.

with knockdown assays using dsRNA (Fig. 2D, lanes 5,7). Similarly, transactivation was attenuated when the known EcR coactivator Tai was knocked down (Fig. 2D, lane 6).

To determine if the observed association of EcR with dDEK also occurred at the EcR target gene promoter, the endogenous promoter of the Eip $75 B$ gene in S2 cells was subjected to chromatin immunoprecipitation (ChIP) analysis. Although the EcR-binding sites remain to be mapped in this promoter, an EcR-binding site was found in the first intron region designated as position $\mathrm{E}$, after testing regions A-J (see Supplemental Fig. S4C). EcR was recruited upon Mur treatment, and recruitment of dDEK was also inducible by Mur at site E (Fig. 2E). When EcR was knocked down in S2 cells, recruitment of dDEK was abolished. In contrast, dDEK was not indispensable for ligand-induced EcR recruitment (Fig. 2E).

\section{Biochemical identification of a dDEK complex}

Regulators reorganizing chromatin's configuration often form nuclear multisubunit complexes. Thus, a dDEK- containing complex was biochemically purified from the nuclear extracts (NEs) of a newly established stable S2 transformant expressing Flag-tagged dDEK (e-dDEK). A dDEK-containing complex was purified through several chromatographic steps (Fig. 3A), and the components were analyzed using MALDI-TOF/mass spectrometry (MS) (Ohtake et al. 2007; Fujiki et al. 2009). The $\alpha$ and $\beta$ subunits of Drosophila CK2 (dCK2) were copurified and identified together with dDEK (Fig. 3B, top panel), and were further verified by Western blot (Fig. 3B, bottom panel). The purified dDEK-CK2 complex [dDEK (NE) com] exhibited a size of $\sim 670 \mathrm{kDa}$ by gel filtration, and dDEK was phosphorylated (Fig. 3C, bottom panel). Consistent with the isolation of the dDEK-CK2 complex, colocalization of $\mathrm{dCK} 2 \alpha$ with $\mathrm{dDEK}$ and $\mathrm{EcR}$ was detected in the puff regions on polytene chromosome (Supplemental Fig. S5A,B).

Functional kinase activity of CK2 is achieved through formation of a heterotetramer containing the catalytic $\alpha$ subunit and the regulatory $\beta$ subunit $(\sim 130 \mathrm{kDa})$ (Litchfield 2003). As bacterially expressed dDEK recombinant proteins 
A
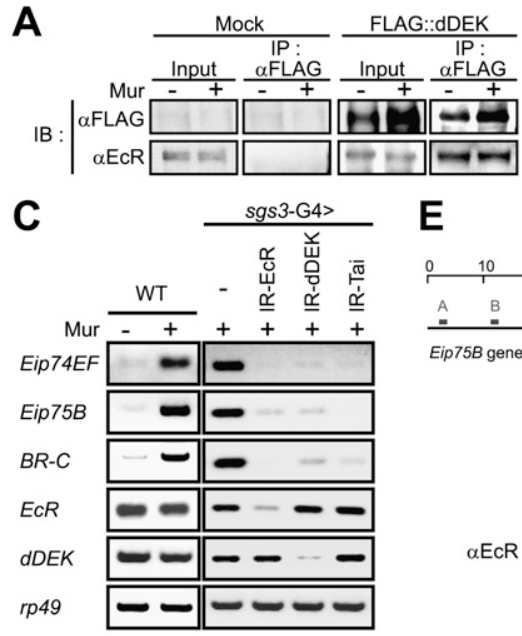

D

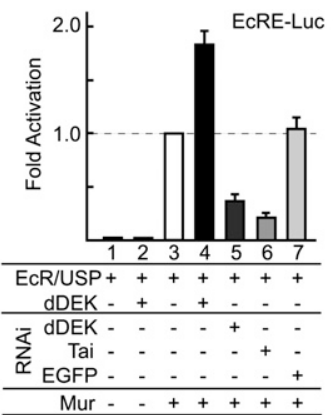

B

E

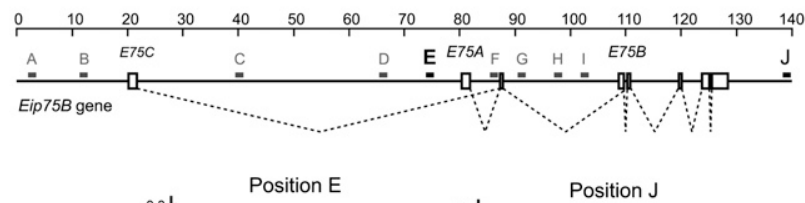

Flow through Pull-down

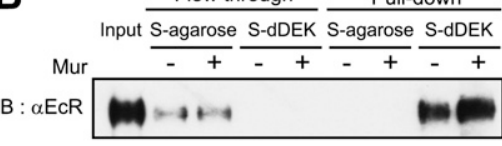

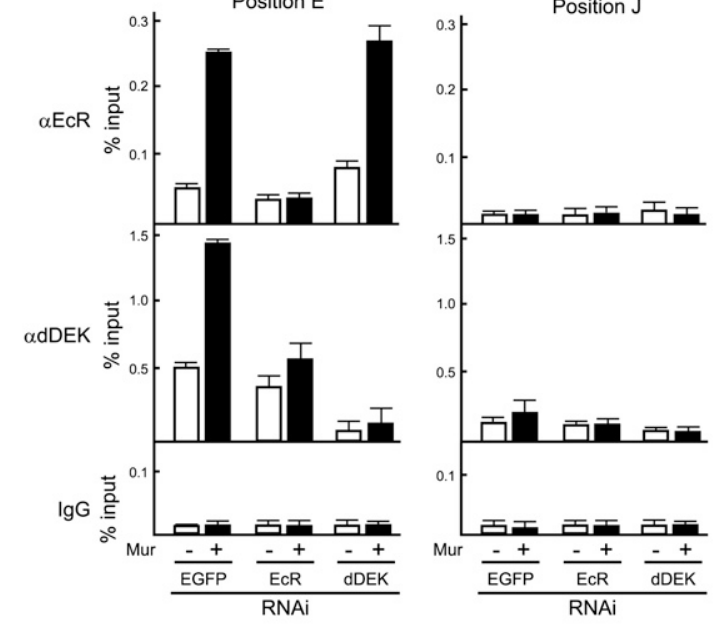

Figure 2. dDEK acts as a coactivator of the EcR. (A) Association of dDEK with EcR in S2 cells. NEs prepared from either S2 cells (Mock) or S2 cells stably expressing Flag-tagged dDEK (e-dDEK) were subjected to immunoprecipitation (IP) with an anti-Flag antibody. EcR was detected by Western blotting using an anti-EcR antibody. $(B)$ Direct interaction of dDEK with EcR in pull-down assay. EcR and USP were incubated with S-tagged dDEK immobilized on beads in the absence or presence of Mur $\left(2.5 \times 10^{-7} \mathrm{M}\right)$. Flowthrough fractions and bound fractions were analyzed by Western blotting using an anti-EcR antibody. $(C)$ Semiquantitative RT-PCR analysis of EcR target genes in third instar larval salivary glands untreated (-) or treated with Mur (+) for $2 \mathrm{~h}$. RT-PCR was performed for Eip74EF, Eip75B, BR-C, EcR, $d D E K$, and $r p 49$ (as a control) in RNA isolated from salivary glands expressing IR-EcR, IR-dDEK, or IR-Tai transgene for RNAi-mediated knockdown. The knockdown efficiencies are shown in Supplemental Figure S3A. $(D)$ Coactivation of liganded EcR function by dDEK in S2 cells. For reporter assays, S2 cells were transfected with indicated plasmid (EcRE-Luc, EcR, and USP with or without $\mathrm{dDEK})$ and then incubated for $16 \mathrm{~h}$ in the absence or presence of Mur $\left(2.5 \times 10^{-7} \mathrm{M}\right)$. For knockdown of endogenous dDEK or Tai (EGFP as a negative control), the cells were preliminarily transfected with dsRNA followed by incubation for $72 \mathrm{~h}$. The knockdown efficiencies are shown in Supplemental Figure S3B. Results are given as means \pm SD of at least three independent experiments. $(E)$ Ligand-dependent recruitment of dDEK with EcR on specific sites of the Eip $75 B$ gene. The map of Eip $75 \mathrm{~B}$ gene region is shown in the top panel. For ChIP analysis, soluble chromatin prepared from S2 cells treated with or without Mur $\left(2.5 \times 10^{-7} \mathrm{M}\right)$ for $1 \mathrm{~h}$ was immunoprecipitated with indicated antibodies. Black bars (E and J) indicate positions of the amplicons for quantitative PCR. The amplicon of position $\mathrm{J}$ is a negative control. In the bottom panel, the values are expressed as a percentage of the amount of immunoprecipitated DNA normalized to respective input DNA signals. Results are given as means \pm SD of at least three independent experiments.

(full-length [FL] and 215-415) also appeared to form a tetramer by Superose 6 gel filtration analysis (Supplemental Fig. S6A), glycerol gradient sedimentation (Supplemental Fig. S6B), and Blue Native PAGE (Supplemental Fig. S6C; Wittig et al. 2006), we assumed that this complex was composed of the two tetramers.

Association of dDEK with the CK2 $\alpha$ subunit was tested in a pull-down assay with dDEK mutants (Fig. 4A; Supplemental Fig. S7). The dDEK SAP domain (282-316 amino acids), a putative DNA-binding motif, was mapped as a CK2 $\alpha$-interacting domain, and this association was potentiated by the presence of ATP (Fig. 4B), suggesting phosphorylation dependency in the interaction between
dDEK and CK2 $\alpha$. Furthermore, formation of the dDEK$\mathrm{CK} 2 \alpha$ and dDEK-CK2 $\beta$ complex was detected as shiftedup bands only in the presence of ATP (Fig. 4C).

We then asked whether CK2 phosphorylated dDEK. The dDEK protein was phosphorylated by affinity-purified dCK $2 \alpha / \beta$ from S2 cells as well as human CK $2 \alpha / \beta$, and this phosphorylation was clearly blocked by a CK2 inhibitor: DMAT (Fig. 4D). The triple mutant (G303A/ S304A/K305A; GSK > AAA), which is clearly defective in its interaction with $\mathrm{CK} 2 \alpha$, was significantly less phosphorylated by CK2 than dDEK(WT) (Fig. 4E). Thus, dDEK appeared to form a complex with CK2 through the phosphorylated dDEK. 
A

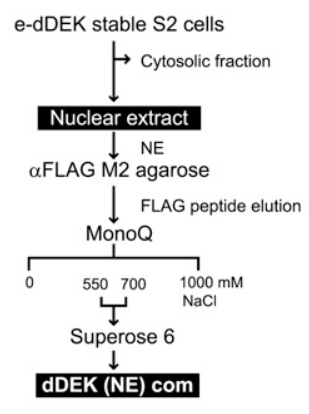

B

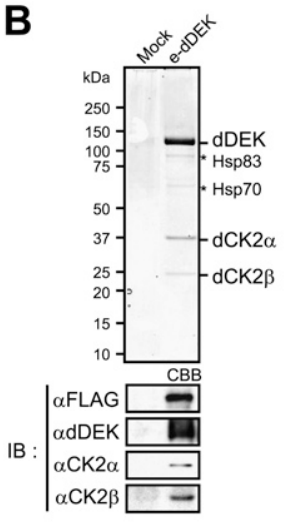

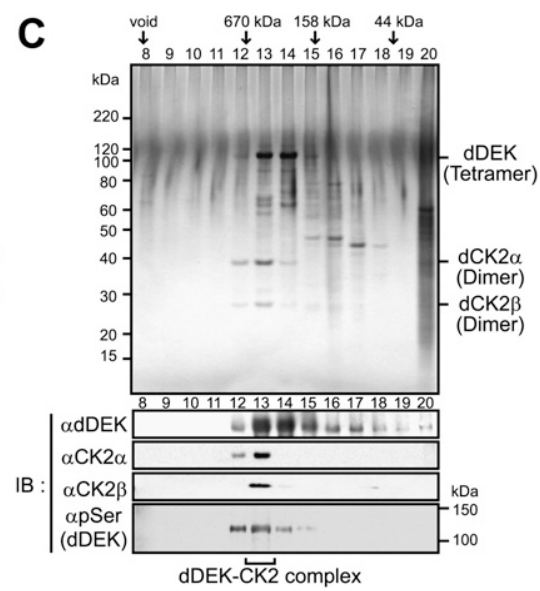

Figure 3. Identification of nuclear dDEK complex containing dCK2. (A) Purification scheme for the dDEK-containing complex in NEs. (B) Identification of the dDEK complex. (Top panel) Coomassie blue staining of the peak fraction of dDEK from the Mono Q purification step after anti-Flag affinity purification for subsequent MALDITOF/MS analysis. Subunits of the complex are identified on the right. Asterisks indicate background proteins. The fractions were analyzed by Western blotting using the indicated antibodies in the bottom panels. $(C)$ Silver staining (top) and Western blot (bottom) analysis of dDEK-containing complex [dDEK (NE) com] fractionated by Superose 6 gel filtration after Mono Q purification. The elution profile of the protein markers is indicated at the top.

\section{dDEK is a CK2-dependent nucleosome assembly factor}

During characterization of the dDEK-CK2 complex, dDEK was found to tightly associate with chromatin, and it copurified with core histones from the soluble chromatin fraction of micrococcal nuclease (MNase)digested nuclear pellets (Supplemental Fig. S8A,B). Immunoprecipitation of dDEK from the chromatin fraction from S2 cells revealed that phosphorylated dDEK associated with core histones (Fig. 5A, left panel), and knockdown of CK2 $\alpha$ in S2 cells resulted in the loss of dDEK complex formation with histones (Fig. 5A, right panel). In a pull-down assay using S-tagged dDEK beads, dDEK interacted directly with native core histones purified from S2 cells, but $\mathrm{CK} 2 \alpha / \beta$ was not needed for this association in vitro (Supplemental Fig. S9). However, when the histones were reconstituted with plasmid DNA, histone association with dDEK-CK2s was abolished (Supplemental Fig. S9). These findings led us to suggest that dDEK serves as a histone chaperone to assemble histones into chromatin.

To test this idea, we asked if dDEK was capable of forming a nucleosomal array. Using a reconstitution assay with native core histones purified from S2 cells and supercoiled plasmid DNA (Supplemental Fig. S10A; Ito et al. 1997), dDEK was found to transfer histones to DNA, resulting in the formation of a histone-DNA complex that was evident in the bottom fraction of the glycerol density gradient (Supplemental Fig. S10B). Furthermore, MNase digestion analysis of this bottom fraction detected formation of mono- and dinucleosomes (Supplemental Fig. S10C). Then, we asked if dDEK could replace a wellknown histone chaperone: Drosophila NAP-1 (dNAP-1). An in vitro nucleosome assembly assay was performed with Drosophila ACF and dNAP-1 proteins as well as core histones (Supplemental Fig. S11) in the presence of plasmid DNA (Ito et al. 1997; Kitagawa et al. 2003). Reconstitution of the nucleosomal array by dNAP-1 histone chaperone and $\mathrm{dACF}$ was confirmed by MNase digestion analysis (Fig. 5B, lanes 3,4). Under these conditions, the purified dDEK-CK2 complex [dDEK (NE) com] transferred core histones to DNA (Fig. 5B, lanes 5,6). Importantly, the chaperone activity of this complex was abolished in the presence of a CK2 inhibitor: DMAT (Fig. 5B, lanes 7,8). The recombinant $\mathrm{dDEK}$ protein alone showed only weak nucleosome assembly activity (Fig. 5C, lanes 5,6), but, as expected, its activity was potentiated by the presence of recombinant human CK2 (hCK2) (Fig. 5C, lanes 7,8). As anticipated, the GSK > AAA mutant had no CK2dependent histone chaperone activity (Fig. 5D, lanes 7,8). Thus, these findings suggest that $\mathrm{DDEK}$ requires CK2 for histone chaperone activity, achieved through formation of a functional complex via phosphorylation of dDEK.

\section{dDEK assembles transcriptionally active chromatin}

We then asked if dDEK preferentially associated with transcriptionally active or inactive histone modifications using a pull-down assay with S-tagged $\mathrm{dDEK}$ and the chromatin fraction from S2 cells (Supplemental Fig. S12A). Consistent with dDEK localization in transcriptionally active regions on polytene chromosomes (Fig. 1C), histones pulled down with dDEK were marked with transcriptionally active modifications such as histone H3K4 methylation (Supplemental Fig. S12B). As dDEK appeared to associate with histones in the transcriptionally active chromatin, we then asked if dDEK also interacted with the histone $\mathrm{H} 3$ variant $\mathrm{H} 3.3$. When Myc-tagged histones were overexpressed in S2 cells, dDEK was efficiently coimmunoprecipitated with H3.3, but much less with $\mathrm{H} 3$ (Fig. 6A). By in vitro binding assays, dDEK exhibited affinity for $\mathrm{H} 2 \mathrm{~A}-\mathrm{H} 2 \mathrm{~B}$ dimers, but the association with the $\mathrm{H} 3.3-\mathrm{H} 4$ tetramer appeared to be more stable (Fig. 6B). To address this point under physiological conditions, H3.3's association with dDEK was tested in intact flies. dDEK's localization pattern in the salivary gland overlapped with that of H3.3, rather than that of $\mathrm{H} 3$ (Fig. 6C). As dDEK physically interacts with 


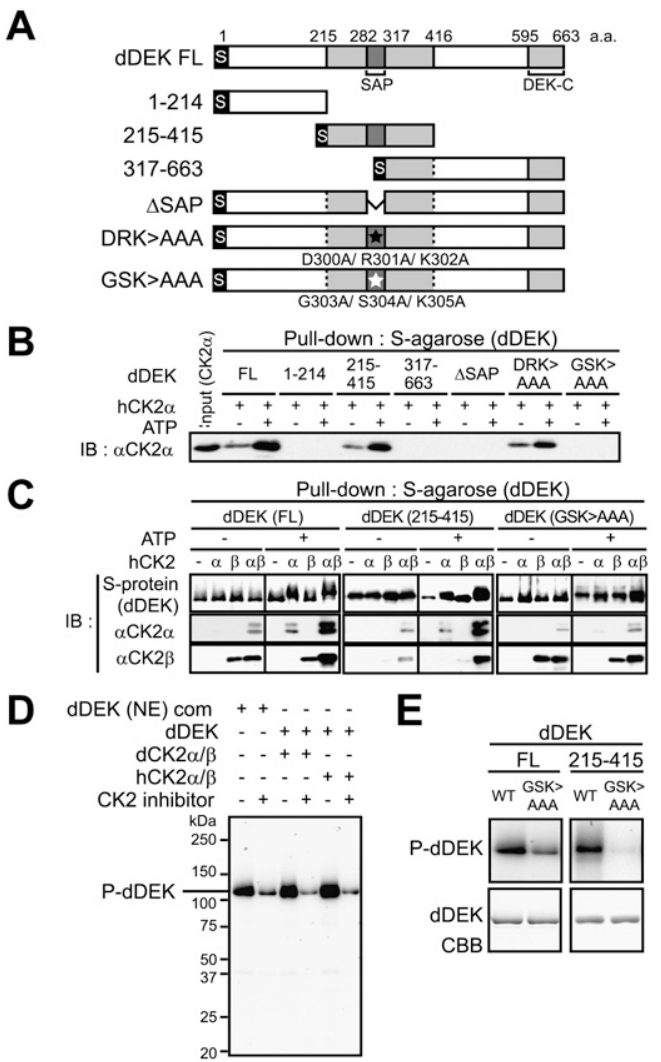

Figure 4. CK2-mediated phosphorylation of dDEK regulates formation of the dDEK-CK2 complex. (A) Schematic representation of dDEK domain organization of full-length (FL) and mutant variants. The gray boxes represent the evolutionarily conserved region containing SAP (pfam 02037) or DEK-C (pfam 08766) domains. For each protein, the location of $\mathrm{N}$-terminal $\mathrm{S}$-tag is shown. Triple-alanine mutated residues are shown as closed asterisks (D300A/R301A/K302A; DRK > AAA) or opened asterisks (G303A/S304A/K305A; GSK > AAA). (B) Determination of the human CK2 $\alpha$ subunit-binding region in dDEK. For pull-down assays, recombinant hCK $2 \alpha(1 \mu \mathrm{g})$ was incubated with S-tagged dDEK $(5 \mu \mathrm{g})$-immobilized beads in the absence or presence of $100 \mu \mathrm{M}$ ATP. The pull-downs were analyzed by Western blotting using anti-CK2 $\alpha$ antibody. $(C)$ Phosphorylationdependent formation of the dDEK-hCK2 complex. Recombinant hCK $2 \alpha(1 \mu \mathrm{g})$ and/or hCK $2 \beta(0.6 \mu \mathrm{g})$ proteins were pulled down by S-tagged FL and mutant dDEK proteins with or without ATP. Bound proteins were analyzed by SDS-PAGE and Western blotting using indicated antibodies. $(D, E)$ Phosphorylation of dDEK by CK2. In vitro kinase assays using ${ }^{32} \mathrm{P}-\gamma$-ATP were performed using purified dDEK (NE) complex or indicated recombinant proteins in the absence or presence of $5 \mu \mathrm{M}$ DMAT as CK2 inhibitor $(D)$, and dDEK mutant variants $(E)$. The phosphorylated products were visualized by autoradiography.

EcR, H3.3's localization was then examined in ecdysoneinduced puff loci in the salivary glands (Schwartz and Ahmad 2005). As a result, H3.3 accumulation and colocalization with dDEK were observed in a Mur-dependent manner on puffs. (Fig. 6D, top panel). However, when the GSK > AAA mutant was expressed, H3.3 accumulation induced by Mur treatment was impaired, regardless of dDEK accumulation in the puff sites (Fig. 6D, bottom panel). Consistent with these observations, the Mur effect in the induction of EcR-target genes was defective in the GSK > AAA mutant (Fig. 6E; Supplemental Fig. S13). In a reporter assay, the GSK > AAA mutant lost the EcR coactivator function, and the CK2 inhibitor attenuated dDEK-mediated coactivation (Supplemental Fig. S14). Thus, it is likely that dDEK coactivated EcR through the assembly of active chromatin, enriched in transcriptionally active histone marks, within EcR target gene promoters.

The nucleosome assembly function of DEK is evolutionarily conserved between flies and humans

While the size of the hDEK protein $(43 \mathrm{kDa})$ is much smaller than that of $\mathrm{dDEK}$, the central and C-terminal regions of hDEK are highly conserved (Fig. 7A). Therefore, we asked if hDEK also served as a histone chaperone. First, hDEK-containing complexes were biochemically purified from a newly established stable HeLa transformant expressing Flag-His ${ }_{6}$-tagged hDEK (e-hDEK) (Fig. 7B). Similar to dDEK, two types of hDEK complexes were identified. One complex contained hCK2 $\alpha / \alpha^{\prime}$ and $\beta$ subunits (Fig. 7C), and core histones were detected in the other fraction (Fig. 7D). By in vitro nucleosome assembly, the purified hDEK-CK2 complex was capable of forming nucleosomes (data not shown). In vitro, recombinant hDEK had significant nucleosome assembly activity in the presence of recombinant $\mathrm{hCK} 2 \alpha / \beta$, and it was attenuated by the CK2 inhibitor DMAT (Fig. 7E). Thus, like dDEK, hDEK appears to be a histone chaperone.

\section{Impaired histone chaperone function in the leukemia $D E K-C A N$ fusion protein}

In a group of AML patients, the human DEK gene (located on chromosome $6 \mathrm{p} 23$ ) is fused to the CAN gene on chromosome $9 \mathrm{q} 34$ by chromosomal translocation (von Lindern et al. 1992). In such AML patients, the C-terminal domain of hDEK is spliced into the $\mathrm{N}$-terminal truncated CAN (Fig. 7A), and malfunction of hDEK has been assumed. To address this issue, the histone chaperone function of hDEK-CAN was assessed. When the hDEKCAN fusion protein was expressed in HEK 293T cells, the association of hDEK with either CK $2 \alpha, \mathrm{CK} 2 \beta$, or histones was abolished (Fig. 7F). In EcR-mediated activation of the endogenous EcR target genes in intact flies, hDEK-CAN acted as a dominant-negative mutant (Fig. 7G; Supplemental Fig. S15). We attribute the disruption of functional complex formed by endogenous DEK-CK2 to hDEK/hDEK-CAN oligomerization (Fig. 7F). Thus, it appears that hDEK fusion to CAN impairs histone chaperone activity and contributes to the onset of this type of AML.

\section{Discussion}

Genetic identification of an EcR coregulator by an EGFP protein trap approach

Puff formation has long been recognized as reconfiguration of chromatin, a change that plays an important 
A

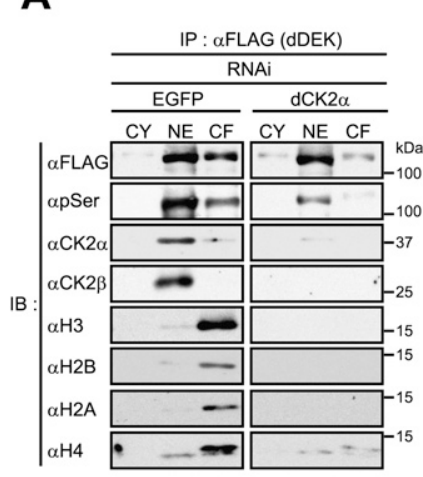

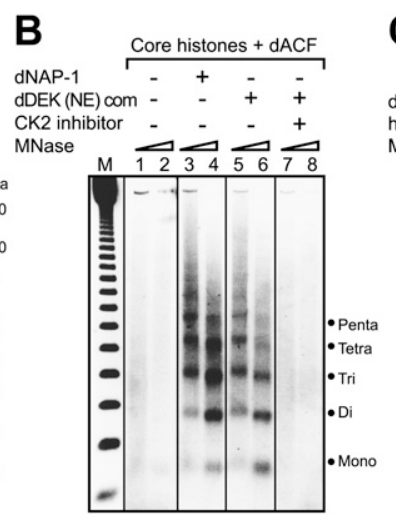
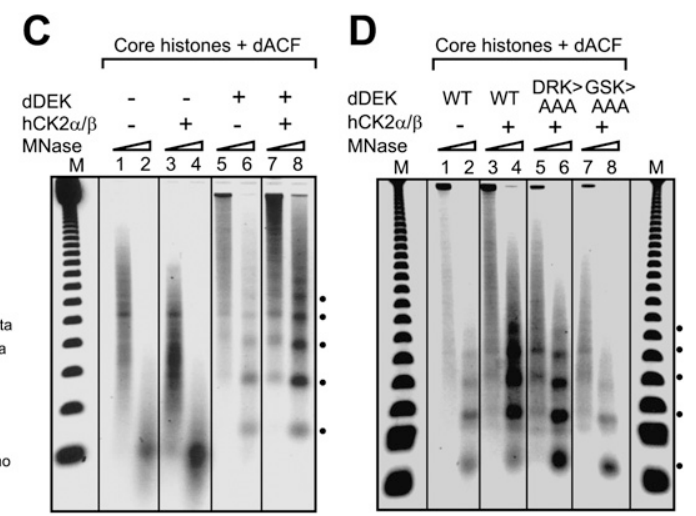

Figure 5. dDEK is a novel nucleosome assembly factor. $(A)$ CK2-mediated phosphorylation of dDEK is required for association with core histones. The cytosolic fraction (CY), NE, and chromatin fraction (CF) derived from S2 cells stably expressing e-dDEK were used for immunoprecipitation using anti-Flag M2 resin. The immunoprecipitates were subjected to Western blot analysis using the indicated antibodies. For knockdown of endogenous $\mathrm{dCK} 2 \alpha$ (right panel), the cells were transfected with dsRNA (EGFP as a negative control, left panel). The knockdown efficiencies are shown in Supplemental Figure S3C. $(B-D)$ In vitro nucleosome assembly assays. $(B)$ Purified dDEK-CK2 complex from NE [DDEK (NE) com] or dNAP-1 (as a positive control) were incubated with purified dACF, core histones, and plasmid DNA in the presence of CK2 inhibitor DMAT or DMSO (control) for $3 \mathrm{~h}$ at $27^{\circ} \mathrm{C}$. The samples were partially digested with varying concentrations of MNase used to digest the chromatin. The resulting DNA fragments were visualized by ethidium bromide staining. The molecular mass markers $(\mathrm{M})$ are the 123-bp ladder. The nucleosome positions are indicated at the right. Recombinant dDEK $(C)$ and dDEK mutants $(D)$ were subjected to the same assay as $B$. dDEK (DRK > AAA) protein is used as a negative control.

physiologic role at the molecular level (Ashburner 1967, 1990). However, factors responsible for the reconfiguration have remained largely unidentified. The steroid hormone ecdysone induces puff formation, and we genetically screened for EcR coregulators. For this purpose, an EGFP protein trap approach was applied to screen for factors colocalized with EcR on polytene chromosomes. Several candidate factors encoded known transcriptional coregulators: osa (a SWI/SNF complex component) (Supplemental Fig. S16) and skd (a mediator complex component) (Boube et al. 2000; Mohrmann et al. 2004; data not shown), as well as functionally uncharacterized proteins, including dDEK. The chromosomal localization revealed that dDEK supports the process of gene activation coupled to puff formation.

\section{$D E K$ is a histone chaperone}

Two forms of DEK complexes were biochemically purified from insect and human cells, with very similar compositions (Figs. 3B, 7C, 7D; Supplemental Fig. S8B). We hypothesize that formation of the dDEK-CK2 complex is required for $\mathrm{dDEK}$ phosphorylation, rendering histone chaperone activity, while the dDEK-histone complex represents an intermediate during assembly into chromatin. Reflecting dDEK localization (Fig. 1C), histones associating with dDEK harbored epigenetic marks for transcriptional activation (Supplemental Fig. S12B). The purified complex contained H3.3 (data not shown), and dDEK appears to accommodate more $\mathrm{H} 3.3-\mathrm{H} 4$ tetramers than H3-H4 in S2 cells (Fig. 6A). Moreover, on polytene chromosomes, we observed that dDEK preferentially colocalized with H3.3 rather than with H3 (Fig. $6 C)$. It is unclear at this stage if $\mathrm{dDEK}$ is also capable of disassembling histones from chromatin. While HIRA has been characterized as a histone chaperone for $\mathrm{H} 3.3$ (Tagami et al. 2004; Henikoff 2008), H3.3 deposition on chromosome has been observed in flies deficient of HIRA (Bonnefoy et al. 2007). dDEK may be functionally similar to HIRA in H3.3 assembly into chromatin. Together with the previous findings that nucleosomes containing H3.3 tend to be less stable than those containing H3 (Jin and Felsenfeld 2007), it is conceivable that dDEK remodels nucleosomal histone octamers into more transcriptionally active octamers through its histone chaperone activity, in cooperation with chromatin remodelers (Henikoff and Ahmad 2005; De Koning et al. 2007; Henikoff 2008). During puff formation, EcR may recruit dDEK to reconfigure the nucleosomal array during chromatin reorganization.

\section{The histone chaperone activity of DEK depends on its phosphorylation by CK2}

In the in vitro chromatin assembly system, DEK function required $\mathrm{CK} 2$, and a CK2 kinase inhibitor abrogated chromatin assembly. The dDEK mutant (GSK > AAA) was unable to stably associate with CK2 $\alpha$ (Fig. 4B,C) and was defective in assembling histones on DNA (Fig. 5D). Thus, CK2 requires the phosphorylation of dDEK to form a dDEK-CK2 complex as a histone chaperone. Like CK2, the DEK-CK2 functional complex appears to be composed of two units: One is a phosphorylated dDEK tetramer functioning as a histone chaperone, and the other is a CK2 tetramer functioning as a regulatory unit. In this respect, DEK appears to be an atypical histone chaperone in terms of phosphorylation dependency as well as subunit composition, although a number of histone chaperones have been described with diverse forms of oligomerization (Park and Luger 2008). 
A

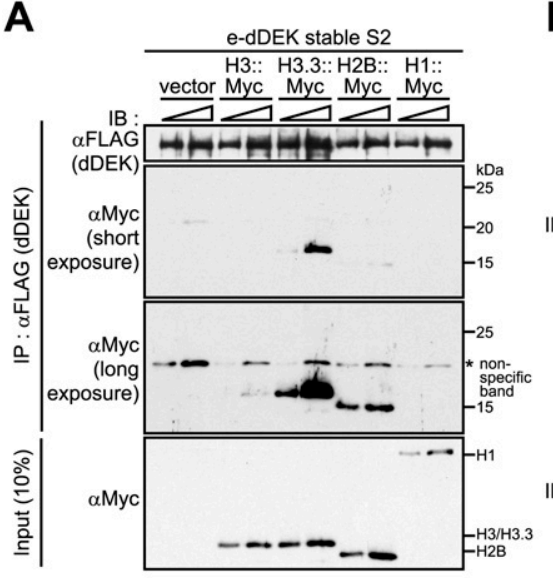

D

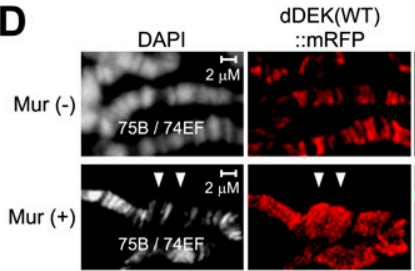

$758 / 7456$

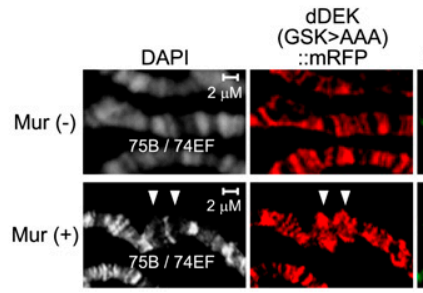

B

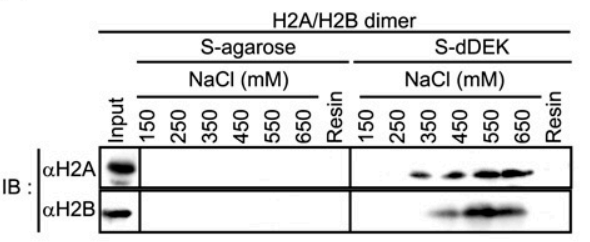

$\mathrm{H} 3.3 / \mathrm{H} 4$ tetramer
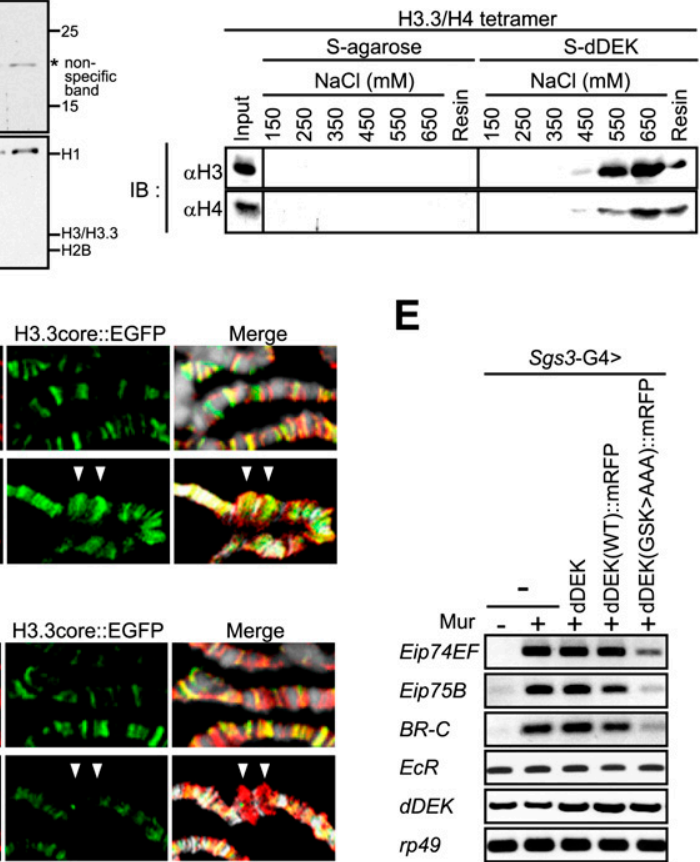

E

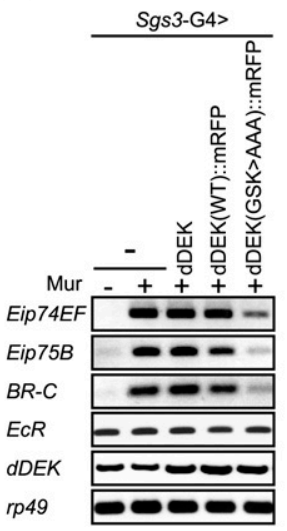

C
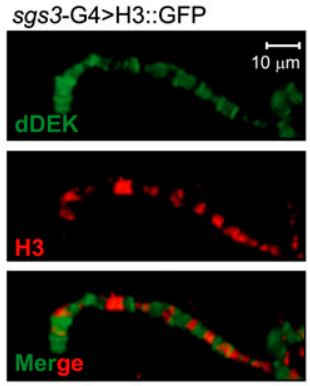

sgs3-G4>H3.3core::GFP
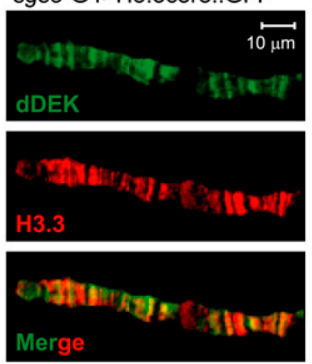

Figure 6. The nucleosomal assembly activity of $\mathrm{dDEK}$ is required for ecdysone-inducible puff formation and EcR target gene expression. (A) Preferential association of dDEK with a histone variant, H3.3. Chromatin fractions from e-dDEK stable cells transfected with H3-myc, H3.3-myc, H2B-myc, or H1-myc were subjected to immunoprecipitation using anti-Flag M2 resin. Each immunoprecipitated histone was detected using anti-Myc antibody. (B) Direct interaction of dDEK with histone H3.3/H4 tetramer. For pull-down assays, reconstituted recombinant Drosophila $\mathrm{H} 2 \mathrm{~A} / \mathrm{H} 2 \mathrm{~B}$ dimer or $\mathrm{H} 3.3 / \mathrm{H} 4$ tetramer were incubated with S-tagged dDEK protein immobilized on S-protein beads. Wash fractions (at each $\mathrm{NaCl}$ concentration) and final elution fractions were analyzed by Western blotting using the indicated antibodies. $(C)$ Colocalization of dDEK with variant H3.3, but not canonical H3. Polytene chromosomes from larvae expressing H3::EGFP (top) or H3.3core::EGFP (bottom) fusion proteins driven by sgs3-GAL4 were coimmunostained with anti-dDEK (green) and anti-GFP (red) antibodies. $(D)$ dDEK-dependent accumulation of $\mathrm{H} 3.3$ at ecdysone-inducible puff loci 74EF and 75B (arrowheads). The salivary glands from larvae coexpressing H3.3core::EGFP (green) with dDEK(WT)::mRFP or dDEK(GSK > AAA):: mRFP (red) fusion proteins driven by sgs3-GAL4 were treated without or with Mur $\left(2.5 \times 10^{-7} \mathrm{M}\right)$ as described in Figure $1 \mathrm{C}$. The polytene chromosomes were subjected to immunostaining with anti-GFP and anti-RFP antibodies. (E) Abrogated expression of EcRtarget genes in the fly line expressing dDEK defective in histone chaperone activity. RT-PCR was performed in RNA isolated from larval salivary glands expressing dDEK, dDEK(WT)::mRFP, or dDEK(GSK > AAA)::mRFP transgene. Semiquantitative RT-PCR analysis was performed as described in Figure 2C.

\section{$D E K$ is a transcriptional coactivator for EcR}

It is believed that histone chaperones coregulate transcription; however, no clear evidence, particularly in intact animals, supports a histone chaperone serving as a transcriptional coregulator for a given transcriptional activator. Here, we show that endogenous dDEK coactivates endogenous EcR in the chromosomal regions of ecdysone-induced puffs in intact flies (Fig. 2C). Its coactivator function is likely attributable to its histone chaperone activity (Fig. 6E; Supplemental Fig. S14), leading to assembly of active histones in chromatin (Fig. 6A; Supplemental Fig. S12B). As dDEK and EcR were detected together with CK2 $\alpha$ at the EcR-binding sites in EcR target genes (Fig. 2E; Supplemental Fig. S5C), they likely contribute to ecdysone-dependent nucleosomal reorganization. In this respect, dDEK may be unique among histone chaperones in terms of its selective and physical interaction with EcR (Fig. 2A,B). However, other histone chaperones may also assist in the assembly and disassembly of histone octamers on promoter/enhancer sequences where EcR regulates gene expression. This concept is supported by data showing ecdysone-induced puff formation was not abrogated in a transgenic fly line overexpressing a dominant-negative form of $\mathrm{dDEK}$ 
A
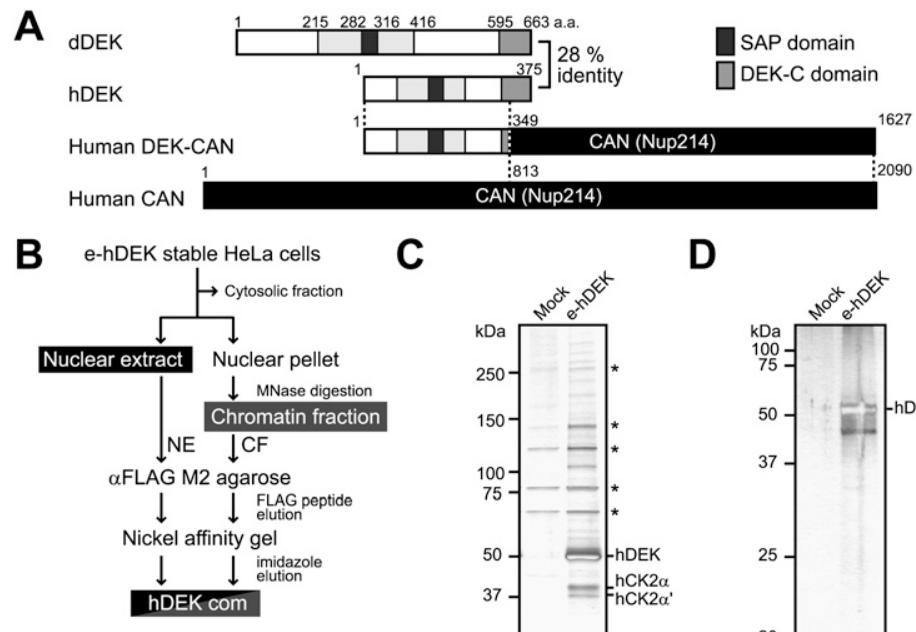

G

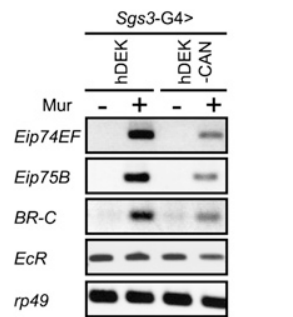

C

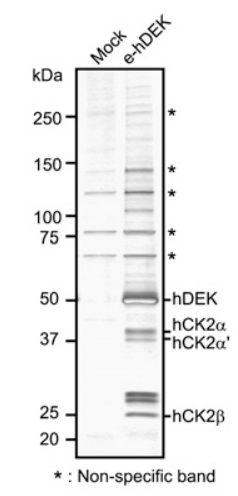

D

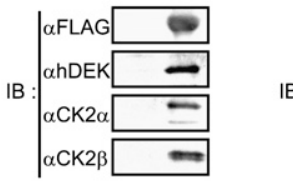

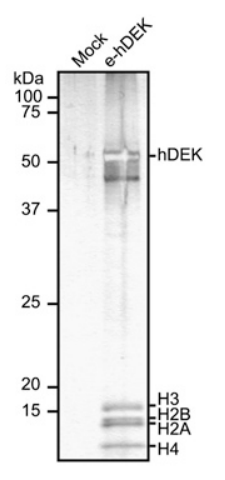

E

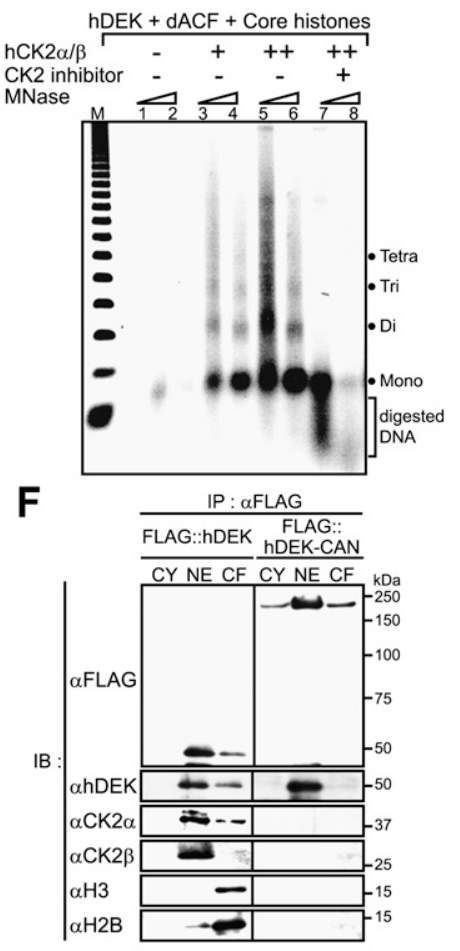

Figure 7. Functional characteristics of hDEK and leukemia-associated fusion protein, DEK-CAN. (A) Schematic representation of dDEK, hDEK, hCAN, and hDEK-CAN fusion proteins (von Lindern et al. 1992). (B) The purification scheme for the hDEK-containing complex. $(C, D)$ Mass spectrometric and Western blot analysis of the hDEK complex components purified from the NE $(C)$ or chromatin fraction $(D)$ in e-hDEK stable HeLa cells. Silver staining of the peak fraction of hDEK from the final Ni affinity purification step is shown in the top panels. MALDI-TOF/MS analysis of the complex subunits is shown on the right. Asterisks indicate background proteins. Purified hDEK complex fractions were used for Western blotting with the indicated antibodies in the bottom panels. (E) Histone chaperone activity of hDEK in vitro. Recombinant hDEK was subjected to the nucleosome assembly assay as described in Figure 5B. (F) Lack of functional components of hDEK complex in hDEK-CAN-overexpressing 293T cells. The cytosolic fraction (CY), $\mathrm{NE}$, and chromatin fraction (CF) derived from 293T cells transiently transfected with Flag-tagged hDEK or hDEK-CAN were used for immunoprecipitation using anti-Flag M2 resin. The immunoprecipitates were subjected to Western blotting with the indicated antibodies. $(G)$ Abrogated expression of EcR target genes in the fly line expressing hDEK-CAN leukemic fusion protein. RT-PCR was performed in RNA isolated from larval salivary glands expressing hDEK or hDEK-CAN. Semiquantitative RT-PCR analysis was performed as described in Figure 2C.

(GSK > AAA) (Fig. 6D), regardless of aberrant expression of the tested EcR target genes (Fig. 6E).

dDEK localization on polytene chromosomes was also seen in regions other than the puff sites, and the localization pattern was not significantly affected by knockdown of EcR (data not shown). Since histone chaperone activity supports both eviction and assembly of histone octamers on chromatin, we speculate that the dDEK histone chaperone might serve as an inhibitor for transcriptional events, depending on $\mathrm{dDEK}$ localization on chromosomes. These facets of dDEK function may account for bidirectional coregulatory roles in transcriptional control; i.e., as an activator in the present study versus a repressor in a previous report (Gamble and Fisher 2007).

\section{A class of AML is attributed to impaired histone chaperone activity of hDEK by fusion with CAN}

The similarities of motif organizations in human and fly DEKs suggest that DEKs serve as a class of histone chaperone. Thus, it is feasible that this hDEK function is compromised by its fusion with CAN in AML patients (Fig. 7A; Soekarman et al. 1992; von Lindern et al. 1992). In the present study, we observed that the hDEK-CAN fusion protein is unable to associate with CK2 (Fig. 7F). As phosphorylation of DEK by CK2 was required for histone chaperone activity, it is most likely that histone chaperone function is impaired in the fusion protein. As this stage, the global roles of hDEK function as a histone chaperone in chromosomal changes remain to be defined. However, from the present observations, the hDEK-CAN fusion protein appears defective, at least in part, in assisting transcriptional regulation of certain genes that might be essential for normal differentiation of hematopoietic cells.

\section{Materials and methods}

For a detailed description of the Materials and Methods, see the Supplemental Material. 


\section{Screening}

Salivary glands of third instar larvae of an EGFP protein trap line from 5-d-old cultures were dissected in phosphate-buffered saline (PBS). After removing as much fat as possible, the organ was cultured for $1 \mathrm{~h}$ at $22^{\circ} \mathrm{C}$ in $300 \mu \mathrm{L}$ of Drosophila SFM (Invitrogen) containing $2.5 \times 10^{-7} \mathrm{M}$ Mur (Wako). After incubation, the glands were washed with PBS and immediately fixed for $20 \mathrm{~min}$ in $4 \%$ paraformaldehyde at room temperature. For immunofluorescence staining, they were incubated with anti-EcR primary antibodies (Ag10.2 and DDA2.7 from Developmental Studies Hybridoma Bank), then with Cy3-conjugated anti-mouse secondary antibody (Jackson ImmunoReserch) and DAPI (Roche) as a counterstain for $1 \mathrm{~h}$ at room temperature. Confocal microscopy was performed on a Zeiss Confocal Laser Scanning System 510, and images were assessed using Adobe Photoshop 7.0 (Adobe).

\section{Immunostaining of polytene chromosome squashes}

Immunostaining of polytene chromosome squashes from third instar larvae were performed as described in Zhao et al. (2009) with some modifications. Detailed information is available in the Supplemental Material.

\section{Fly strains}

Fly stocks were maintained at $22^{\circ} \mathrm{C}$ on standard cornmeal-agaryeast food. The yw strain was used as wild-type in all experiments. G00131 and CA06616 (kind gifts from L. Cooley and A. Spradling) were generated by mobilizing the EGFP-trapping P-element as described (Morin et al. 2001; Buszczak et al. 2007). The fly lines expressing Flag-tagged or monomeric RFP (mRFP)tagged dDEK, hDEK, hDEK-CAN, and dCK $2 \alpha$ were created by transforming Drosophila germline cells (BestGene, Inc.) using the pUAST vectors. Several independent lines were established and crossed with sgs3-GAL4 driver lines (sgs3-G4) expressed in salivary glands. RNAi transgenic fly lines of dDEK were obtained using the inducible RNAi method. A 661-base-pair (bp) cDNA fragment (nucleotide position 1-661 of the coding sequence) was amplified by PCR and inserted as an IR in the pUAST-R57 vector (provided by K. Saigo) (Pili-Floury et al. 2004). Knockdown flies were obtained by mating sgs3-G4 to each of the UAS-IR lines. $U A S-I R-E c R$ was obtained from Bloomington Stock Center. UASIR-Tai was provided from R. Ueda. UAS-H3.3core::EGFP and $U A S-H 3:: E G F P$ lines were generous gifts from $\mathrm{K}$. Ahmad (Schwartz and Ahmad 2005).

\section{Purification of dDEK and hDEK complexes}

Drosophila S2 cells stably expressing N-terminal Flag epitopetagged DEK (e-dDEK) were established with a Drosophila Expression System (Invitrogen). The dDEK complex was immunoprecipitated from NEs $(>0.1 \mathrm{~g})$ with anti-Flag $\mathrm{M} 2$ resin in buffer $\mathrm{D}$ (20 mM HEPES-KOH, $0.2 \mathrm{mM}$ EDTA, $5 \mathrm{mM} \mathrm{MgCl}_{2}, 150 \mathrm{mM}$ $\mathrm{KCl}, 0.05 \%[\mathrm{v} / \mathrm{v}] \mathrm{NP}-40,10 \%[\mathrm{v} / \mathrm{v}]$ glycerol, $0.5 \mathrm{mM} \mathrm{DTT}$, 0.2 mM PMSF, protease inhibitor cocktail [Roche] at $\mathrm{pH} 7.9$ ). The bound polypeptides were eluted with the Flag peptide $(0.2 \mathrm{mg} / \mathrm{mL})$ and were subjected to Mono Q chromatography using AKTAexplorer 10S (GE Healthcare) (Fujiki et al. 2009). The components were identified by peptide mass fingerprint analysis using MALDI-TOF/MS (Bruker Daltonics) (Ohtake et al. 2007; Fujiki et al. 2009). For gel filtration, the fractions from the antiFlag purification were loaded onto a Superose 6 10/300 GL column and fractionated with buffer $\mathrm{D}$ in $1-\mathrm{mL}$ fractions using AKTAexplorer 10S.
Nucleosome assembly and MNase digestion assays

Nucleosome assembly and MNase digestion assays were performed as described previously (Ito et al. 1997; Kitagawa et al. 2003). A standard reaction contained supercoiled plasmid DNA, purified core histones from S2 cells, purified recombinant dDEK, purified recombinant dACF, ATP $(3 \mathrm{mM})$, and the ATP-regenerating system.

\section{Acknowledgments}

We thank L. Cooley, A. Spradling, K. Ahmad, D. Montell, T. Tabata, R. Ueda, and K. Saigo for experimental materials. We also appreciate the constructive discussions with H. Kitagawa, F. Ohtake, M. Okada, H. Matsukawa, and S. Takezawa. We extend thanks to Y. Fukuda and T. Suzuki for technical support, and K. Motoi for manuscript preparation. The anti-EcR monoclonal antibody was obtained from the Developmental Studies Hybridoma Bank, developed under the auspices of the NICHD, and maintained by The University of Iowa. This work was supported in part by priority areas from the Ministry of Education, Culture, Sports, Science, and Technology of Japan (to K.T. and S.K.), and by the Kato Nuclear Complex Project grant from the Exploratory Research for Advanced Technology (ERATO), Japan Science and Technology Agency (JST) (to S.K.).

\section{References}

Adkins MW, Tyler JK. 2006. Transcriptional activators are dispensable for transcription in the absence of Spt6-mediated chromatin reassembly of promoter regions. Mol Cell 21: 405-416.

Adkins MW, Howar SR, Tyler JK. 2004. Chromatin disassembly mediated by the histone chaperone Asf1 is essential for transcriptional activation of the yeast $\mathrm{PHO} 5$ and $\mathrm{PHO} 8$ genes. Mol Cell 14: 657-666.

Ashburner M. 1967. Patterns of puffing activity in the salivary gland chromosomes of Drosophila. I. Autosomal puffing patterns in a laboratory stock of Drosophila melanogaster. Chromosoma 21: 398-428.

Ashburner M. 1990. Puffs, genes, and hormones revisited. Cell 61: 1-3.

Bai J, Uehara Y, Montell DJ. 2000. Regulation of invasive cell behavior by taiman, a Drosophila protein related to AIB1, a steroid receptor coactivator amplified in breast cancer. Cell 103: 1047-1058.

Bonnefoy E, Orsi GA, Couble P, Loppin B. 2007. The essential role of Drosophila HIRA for de novo assembly of paternal chromatin at fertilization. PLoS Genet 3: 1991-2006.

Borrelli E, Nestler EJ, Allis CD, Sassone-Corsi P. 2008. Decoding the epigenetic language of neuronal plasticity. Neuron $\mathbf{6 0}$ : 961-974.

Boube M, Faucher C, Joulia L, Cribbs DL, Bourbon HM. 2000. Drosophila homologs of transcriptional mediator complex subunits are required for adult cell and segment identity specification. Genes \& Dev 14: 2906-2917.

Burtis KC, Thummel CS, Jones CW, Karim FD, Hogness DS. 1990. The Drosophila 74EF early puff contains E74, a complex ecdysone-inducible gene that encodes two ets-related proteins. Cell 61: 85-99.

Buszczak M, Paterno S, Lighthouse D, Bachman J, Planck J, Owen S, Skora AD, Nystul TG, Ohlstein B, Allen A, et al. 2007. The carnegie protein trap library: A versatile tool for Drosophila developmental studies. Genetics 175: 15051531.

Cairns BR. 2009. The logic of chromatin architecture and remodelling at promoters. Nature 461: 193-198. 
De Koning L, Corpet A, Haber JE, Almouzni G. 2007. Histone chaperones: An escort network regulating histone traffic. Nat Struct Mol Biol 14: 997-1007.

Evans RM. 1988. The steroid and thyroid hormone receptor superfamily. Science 240: 889-895.

Fujiki R, Chikanishi T, Hashiba W, Ito H, Takada I, Roeder RG, Kitagawa H, Kato S. 2009. GlcNAcylation of a histone methyltransferase in retinoic-acid-induced granulopoiesis. Nature 459: 455-459.

Gamble MJ, Fisher RP. 2007. SET and PARP1 remove DEK from chromatin to permit access by the transcription machinery. Nat Struct Mol Biol 14: 548-555.

Green S, Chambon P. 1988. Nuclear receptors enhance our understanding of transcription regulation. Trends Genet 4: 309-314.

Henikoff S. 2008. Nucleosome destabilization in the epigenetic regulation of gene expression. Nat Rev Genet 9: 1526.

Henikoff S, Ahmad K. 2005. Assembly of variant histones into chromatin. Annu Rev Cell Dev Biol 21: 133-153.

Ito T, Bulger M, Pazin MJ, Kobayashi R, Kadonaga JT. 1997. ACF, an ISWI-containing and ATP-utilizing chromatin assembly and remodeling factor. Cell 90: 145-155.

Jiang C, Pugh BF. 2009. Nucleosome positioning and gene regulation: Advances through genomics. Nat Rev Genet 10: 161-172.

Jin C, Felsenfeld G. 2007. Nucleosome stability mediated by histone variants H3.3 and H2A.Z. Genes \& Dev 21: 15191529.

Karim FD, Thummel CS. 1992. Temporal coordination of regulatory gene expression by the steroid hormone ecdysone. EMBO J 11: 4083-4093.

Kim MY, Mauro S, Gevry N, Lis JT, Kraus WL. 2004. NAD+dependent modulation of chromatin structure and transcription by nucleosome binding properties of PARP-1. Cell 119: 803-814.

King-Jones K, Thummel CS. 2005. Nuclear receptors-a perspective from Drosophila. Nat Rev Genet 6: 311-323.

Kitagawa H, Fujiki R, Yoshimura K, Mezaki Y, Uematsu Y, Matsui D, Ogawa S, Unno K, Okubo M, Tokita A, et al. 2003. The chromatin-remodeling complex WINAC targets a nuclear receptor to promoters and is impaired in Williams syndrome. Cell 113: 905-917.

Koelle MR, Talbot WS, Segraves WA, Bender MT, Cherbas P, Hogness DS. 1991. The Drosophila EcR gene encodes an ecdysone receptor, a new member of the steroid receptor superfamily. Cell 67: 59-77.

Kouzarides T. 2007. Chromatin modifications and their function. Cell 128: 693-705.

Litchfield DW. 2003. Protein kinase CK2: Structure, regulation and role in cellular decisions of life and death. Biochem J 369: $1-15$.

Mito Y, Henikoff JG, Henikoff S. 2005. Genome-scale profiling of histone H3.3 replacement patterns. Nat Genet 37: 10901097.

Mohrmann L, Langenberg K, Krijgsveld J, Kal AJ, Heck AJ, Verrijzer CP. 2004. Differential targeting of two distinct SWI/ SNF-related Drosophila chromatin-remodeling complexes. Mol Cell Biol 24: 3077-3088.

Morin X, Daneman R, Zavortink M, Chia W. 2001. A protein trap strategy to detect GFP-tagged proteins expressed from their endogenous loci in Drosophila. Proc Natl Acad Sci 98: 15050-15055.

Morrison AJ, Shen X. 2009. Chromatin remodelling beyond transcription: The INO80 and SWR1 complexes. Nat ReV Mol Cell Biol 10: 373-384.
Narlikar GJ, Fan HY, Kingston RE. 2002. Cooperation between complexes that regulate chromatin structure and transcription. Cell 108: 475-487.

Ohtake F, Baba A, Takada I, Okada M, Iwasaki K, Miki H, Takahashi S, Kouzmenko A, Nohara K, Chiba T, et al. 2007. Dioxin receptor is a ligand-dependent E3 ubiquitin ligase. Nature 446: 562-566.

Park YJ, Luger K. 2008. Histone chaperones in nucleosome eviction and histone exchange. Curr Opin Struct Biol 18: 282-289.

Pili-Floury S, Leulier F, Takahashi K, Saigo K, Samain E, Ueda R, Lemaitre B. 2004. In vivo RNA interference analysis reveals an unexpected role for GNBP1 in the defense against Grampositive bacterial infection in Drosophila adults. $J$ Biol Chem 279: $12848-12853$.

Roeder RG. 2005. Transcriptional regulation and the role of diverse coactivators in animal cells. FEBS Lett 579: 909915.

Rosenfeld MG, Lunyak VV, Glass CK. 2006. Sensors and signals: A coactivator/corepressor/epigenetic code for integrating signal-dependent programs of transcriptional response. Genes \& Dev 20: 1405-1428.

Sawatsubashi S, Maki A, Ito S, Shirode Y, Suzuki E, Zhao Y, Yamagata K, Kouzmenko A, Takeyama K, Kato S. 2004. Ecdysone receptor-dependent gene regulation mediates histone poly(ADP-ribosyl)ation. Biochem Biophys Res Commun 320: 268-272.

Schwartz BE, Ahmad K. 2005. Transcriptional activation triggers deposition and removal of the histone variant H3.3. Genes \& Dev 19: 804-814.

Sedkov Y, Cho E, Petruk S, Cherbas L, Smith ST, Jones RS, Cherbas P, Canaani E, Jaynes JB, Mazo A. 2003. Methylation at lysine 4 of histone $\mathrm{H} 3$ in ecdysone-dependent development of Drosophila. Nature 426: 78-83.

Sims RJ III, Reinberg D. 2008. Is there a code embedded in proteins that is based on post-translational modifications? Nat Rev Mol Cell Biol 9: 815-820.

Soekarman D, von Lindern M, Daenen S, de Jong B, Fonatsch C, Heinze B, Bartram C, Hagemeijer A, Grosveld G. 1992. The translocation $(6 ; 9)(\mathrm{p} 23 ; \mathrm{q} 34)$ shows consistent rearrangement of two genes and defines a myeloproliferative disorder with specific clinical features. Blood 79: 2990-2997.

Stabell M, Larsson J, Aalen RB, Lambertsson A. 2007. Drosophila dSet2 functions in $\mathrm{H} 3-\mathrm{K} 36$ methylation and is required for development. Biochem Biophys Res Commun 359: 784 789.

Strahl BD, Allis CD. 2000. The language of covalent histone modifications. Nature 403: 41-45.

Tagami H, Ray-Gallet D, Almouzni G, Nakatani Y. 2004. Histone H3.1 and H3.3 complexes mediate nucleosome assembly pathways dependent or independent of DNA synthesis. Cell 116: 51-61.

Takeyama K, Ito S, Yamamoto A, Tanimoto H, Furutani T, Kanuka H, Miura M, Tabata T, Kato S. 2002. Androgendependent neurodegeneration by polyglutamine-expanded human androgen receptor in Drosophila. Neuron 35: 855864.

Thummel CS. 1996. Files on steroids-Drosophila metamorphosis and the mechanisms of steroid hormone action. Trends Genet 12: 306-310.

Thummel CS. 2002. Ecdysone-regulated puff genes 2000. Insect Biochem Mol Biol 32: 113-120.

von Lindern M, Fornerod M, van Baal S, Jaegle M, de Wit T, Buijs A, Grosveld G. 1992. The translocation (6;9), associated with a specific subtype of acute myeloid leukemia, results in the fusion of two genes, dek and can, and the expression of 
Sawatsubashi et al.

a chimeric, leukemia-specific dek-can mRNA. Mol Cell Biol 12: $1687-1697$.

Weeks JR, Hardin SE, Shen J, Lee JM, Greenleaf AL. 1993. Locusspecific variation in phosphorylation state of RNA polymerase II in vivo: Correlations with gene activity and transcript processing. Genes \& Dev 7: 2329-2344.

Wittig I, Braun HP, Schagger H. 2006. Blue native PAGE. Nat Protoc 1: 418-428.

Zhao Y, Takeyama K, Sawatsubashi S, Ito S, Suzuki E, Yamagata K, Tanabe M, Kimura S, Fujiyama S, Ueda T, et al. 2009. Corepressive action of CBP on androgen receptor transactivation in pericentric heterochromatin in a Drosophila experimental model system. Mol Cell Biol 29: 1017-1034. 


\section{Erratum}

Genes \& Development 24: 159-170 (2010)

\section{A histone chaperone, DEK, transcriptionally coactivates a nuclear receptor} Shun Sawatsubashi, Takuya Murata, Jinseon Lim, Ryoji Fujiki, Saya Ito, Eriko Suzuki, Masahiko Tanabe, Yue Zhao, Shuhei Kimura, Sally Fujiyama, Takashi Ueda, Daiki Umetsu, Takashi Ito, Ken-ichi Takeyama, and Shigeaki Kato

Due to an error during figure preparation for the above-mentioned article, one of the images in Figure 2A was accidentally used such that the two top panels, showing negative controls represented as "Mock" (for $\alpha$ Flag), were duplicated. A corrected version of the two panels is shown below, where "IP: $\alpha$ Flag" remains as originally presented and "Input" is replaced by the correct, nonduplicated image. This correction does not alter the conclusions of the study. The authors apologize for the error.

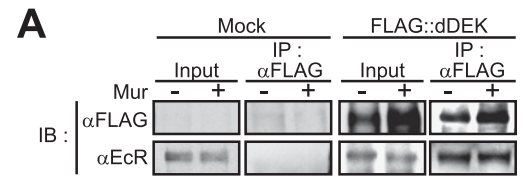




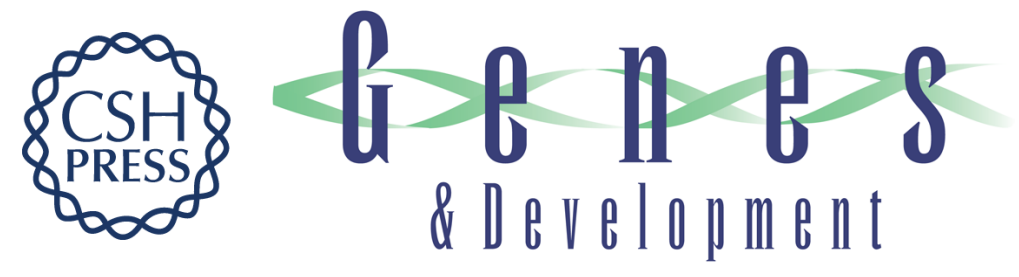

\section{A histone chaperone, DEK, transcriptionally coactivates a nuclear receptor}

Shun Sawatsubashi, Takuya Murata, Jinseon Lim, et al.

Genes Dev. 2010, 24: originally published online December 29, 2009

Access the most recent version at doi:10.1101/gad.1857410

\section{Supplemental http://genesdev.cshlp.org/content/suppl/2009/12/22/gad.1857410.DC1 \\ Material}

Related Content New chaps in the histone chaperone arena

Eric I. Campos and Danny Reinberg

Genes Dev. July , 2010 24: 1334-1338 A histone chaperone, DEK, transcriptionally

coactivates a nuclear receptor

Shun Sawatsubashi, Takuya Murata, Jinseon Lim, et al.

Genes Dev. September , 2012 26: 2118

References This article cites 53 articles, 12 of which can be accessed free at:

http://genesdev.cshlp.org/content/24/2/159.full.html\#ref-list-1

Articles cited in:

http://genesdev.cshlp.org/content/24/2/159.full.html\#related-urls

License

Email Alerting Receive free email alerts when new articles cite this article - sign up in the box at the top Service right corner of the article or click here.

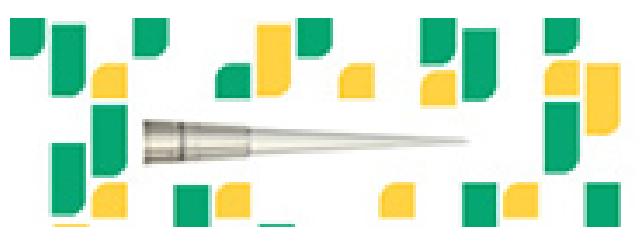

Focused on your science. 